\title{
Modeling Recruitment Maneuvers with a Variable Compliance Model for Pressure Controlled Ventilation
}

\author{
P.S. CROOKE ${ }^{\mathrm{a}, *}$, J.J. MARINI ${ }^{\mathrm{b}, \dagger}$ and J.R. HOTCHKISS ${ }^{\mathrm{b}, \ddagger}$ \\ ${ }^{\mathrm{a}}$ Department of Mathematics, Vanderbilt University, Nashville, TN 37240, USA; ${ }^{\mathrm{b}}$ Pulmonary and Critical Care Medicine, Regions Hospital and
} University of Minnesota, St. Paul, MN 55101, USA

(Received 1 June 2001; In final form 31 July 2002)

\begin{abstract}
A nonlinear mathematical model for pressure controlled, variable compliance ventilation is proposed, solved, and analyzed. The effect of the variable compliance on the key outcome variables, tidal volume, average lung volume, total PEEP, and mean alveolar pressure, is presented. Using human data, the variable compliance model is used to investigate effects of recruitment maneuvers on the key outcome variables.
\end{abstract}

Keywords: Pressure controlled ventilation; Variable compliance; Recruitment; Nonlinear model

\section{NOMENCLATURE}

$V_{i}(t) \quad$ Inspiratory lung volume, $0 \leq t \leq t_{i}$

$V_{e}(t) \quad$ Expiratory lung volume, $t_{i} \leq t \leq t_{\mathrm{tot}}$

$V_{T} \quad$ Tidal volume

$V_{\text {ave }} \quad$ Average lung volume over one breath

$P_{\mathrm{ex}} \quad$ End-expiratory pressure

$V_{\text {ex }} \quad$ End-expiratory volume

$P_{m} \quad$ Mean alveolar pressure

$t_{i} \quad$ Inspiratory time

$t_{e} \quad$ Expiratory time

$t_{\text {tot }} \quad$ Length of one breath

$t_{i_{j}} \quad j=1,2$. Times of compliance changes during inspiration

$t_{e_{j}} \quad j=1,2$. Times of compliance changes during expiration

$f \quad$ Number of breaths per minute

$D \quad$ Inspiratory time fraction

$P_{\text {peep }} \quad$ Applied PEEP during expiration

$P_{\text {set }} \quad$ Applied ventilator pressure

\section{INTRODUCTION}

Numerous mathematical models for the mechanical ventilation of patients with acute respiratory failure have previously been presented in the medical and scientific

\author{
$R_{i} \quad$ Inspiratory resistance \\ $R_{e} \quad$ Expiratory resistance \\ $C_{\text {ave }} \quad$ Average compliance over one breath \\ $C_{i}(V)$ Compliance function during inspiration \\ $C_{e}(V)$ Compliance function during expiration \\ $a_{i_{j}} \quad j=1,2,3$. Compliance parameters during \\ inspiration \\ $b_{i_{j}} \quad j=1,3$. Compliance parameters during \\ inspiration \\ $a_{e_{j}} \quad j=1,2,3$. Compliance parameters during \\ expiration \\ $b_{e_{j}} \quad j=1,3$. Compliance parameters during \\ expiration \\ $V_{i_{j}}^{s} \quad j=1,2$. Volumes at which compliance \\ function changes form during inspiration \\ $V_{e_{j}}^{s} \quad j=1,2$. Volumes at which compliance \\ function changes form during expiration
}

literature (Wald et al., 1968; Barbini, 1982; Marini et al., 1989; Burke et al., 1993; Chandra et al., 1993; Crooke and Marini, 1993; Marini and Crooke, 1993; Hotchkiss et al., 1994; 1996; Morgenstern and Kaiser, 1995; Crooke et al., 1996). The mathematical models range from

\footnotetext{
*Corresponding author.

Supported in part by Health Partners Research Foundation.

${ }^{\ddagger}$ Scientist Development Grantee of the American Heart Association.
} 
one-, two-, or multi-compartment models, permit different resistances for inspiration and expiration, incorporate PEEP, and cover the basic clinical modes of ventilation. However, all of these models have assumed that the compartment (lung) compliance is constant during inspiration and expiration, often with the same compliance. The need of new models for volume dependent compliance as a diagnostic technique in ARDS patient has been advocate in Mols et al. (1999). In this paper, we propose a mathematical model for one-compartment, pressure preset ventilation that incorporates a variable compliance.

Despite its conceptual simplicity and great success in predicting gross behaviors of the pulmonary system in response to imposed pressures or flows, a unicompartmental, linear model of the respiratory system is clearly an oversimplification. Recent data, including that of Jonson and Svantesson (1999), has identified regions of differing compliance characteristics, with lower and upper inflection points, above and below which the compliance behavior of the lung differs. The work of Gattioni et al. (1995) suggests that recruitment of dependent and collapsed airspaces is in large part responsible for the phenomenon of the lower inflection point (LIP). Solway et al. (see for e.g. Yap et al., 1994) have highlighted the potential role of small airway collapse in restricting gas access to particular alveoli. Airspace overdistension is felt likely the underlying cause of the upper inflection point (UIP). Recent data (see for e.g. Hickling, 1998; 2001) demonstrates that regional overdistention may occur at volumes lower than the upper inflection point that is identified from pressure-volume curves obtained at the airway opening. Laboratory studies of Mols (Mols et al., 2001) have shown that the volume-dependent alterations of the lung compliance during mechanical ventilation with associated PEEP levels to maintain alveolar recruitment have a marked impact on pulmonary gas exchange. However, in some cases, a simple recruitment maneuver may not lead to better oxygenation (Fujino et al., 2001). In a recent paper (Villagra et al., 2002), recruitment maneuvers in 17 patients with acute respiratory distress syndrome (ARDS) were studied. These researchers concluded that recruitment maneuvers for ARDS patient have no short-term benefit on oxygenation and may even lead to regional alveolar over distension which in turn may result in the redistribution of blood flow in the lung. Hence, the evidence on the benefits of recruitment maneuvers is mixed. This raises the question: Is there a way for the clinician to know when it is suitable and when it is not? In this paper, we hope to provide a starting point for an answer to this question by providing a mathematical model that is capable of providing a mechanical description of what happens preand post-recruitment maneuver. This will not immediately link the oxygenation with the maneuvers, but it may serve as a mechanical proxy for this important clinical variable.
Nonlinear elastic pressure versus volume curves have been studied extensively in recent years (Silva et al., 1992; Servillo et al., 1997; Nikischin et al., 1998; Svantesson et al., 1998; Venegas et al., 1998; Jonson and Svantesson, 1999; Jonson et al., 1999). If one assumes that the elastic pressure of the lungs, $P_{\text {elastic}}$, is proportional to the lung volume, $V$, then $P_{\text {elastic }}=k V$. If we take $k$ to be a constant, then $1 / k$ is called the lung compliance, $C$. A plot of the elastic pressure versus the volume produces a straight line in the $P_{\text {elastic }}-V$ plane and hence, it yields a linear relationship between these two quantities. However, if we assume that $k$ and hence $C$, depends on $V$, then a plot of elastic pressure versus volume produces a nonlinear relationship. The $P_{\text {elastic }}-V$ curves produced by the nonlinear models are sigmoidal in shape. Often the case, the nonlinear relationship between elastic pressure and volume differs between inspiration and expiration (see for e.g. Suki et al., 1998; Jonson and Svantesson, 1999). The explanation for the sigmoidal shape is that during low lung volumes, the compliance is low, but increasing, due to opening of collapsed lung units. At a particular volume (which in this paper is denoted by $V_{i_{1}}^{s}$ or in the medical literature by $V_{\text {LIP }}$ ), a transition region is entered where the compliance is constant. A third stage is entered at a higher volume $\left(V_{i_{2}}^{s}\right.$ or $\left.V_{\text {UIP }}\right)$ where the compliance is actually decreasing. The sequence of events is reversed during expiration. The shape of the $P_{\text {elastic }}-V$ curves is affected by disease (see e.g. Jonson and Svantesson, 1999) and can be shifted by recruitment maneuvers (Hickling, 1998; Svantesson et al., 1998). To reduce ventilator-induced lung damage, it is often recommended that the ventilation be within the second segment (flat section) of the compliance function. As we will see in the human data presented, recruitment maneuvers can result in a shift in the compliance function ( $V_{i_{1}}^{s}$ and $V_{i_{2}}^{s}$ becomes smaller) and can produce lower tidal volumes, lower average lung volumes, higher end-expiratory pressures, and lower mean alveolar pressures for a given setting of airway pressures $\left(P_{\text {set }}\right.$ and $\left.P_{\text {peep }}\right)$ that are applied by the ventilator during inspiration and expiration.

\section{THE BASIC ONE-SEGMENT MODEL}

The mathematical model for pressure controlled ventilation (PCV) incorporates a pressure that is applied to a single compartment (lung) with a compliance function $C$, inspiratory resistance $R_{i}$, and expiratory resistance $R_{e}$. The ventilator cycle is split into two parts: inspiration of duration $t_{i}$ and expiration of duration $t_{e}$. The total length of each cycle is $t_{\text {tot }}=$ $t_{i}+t_{e}$. During inspiration, a preset pressure, denoted by $P_{\text {set }}$, is applied to the airway and during expiration, the ventilator applies a constant pressure, denoted by $P_{\text {peep}}$, which may be zero. We assume that each new breath is identical to the previous breath. For the flow into and out of the lung we assume that it is equal to the instantaneous rate of change of the volume of the lung. 
In this model, the symbol, $V(t)$, represents the volume above the volume of the lung at the end of the previous breath. Hence, $V(t)$ is referenced to a constant volume $V_{\text {ex }}$ which is explained below. Assuming breaths of uniform length $\left(t_{\text {tot }}\right)$, this means that $V$ is zero at the beginning and ending of each breath. We consider the pressure balances in the system:

$$
P_{\text {resistive }}+P_{\text {elastic }}+P_{\text {ex }}=P_{\text {vent }} .
$$

The term $P_{\text {ex }}$ is called the end-expiratory pressure and represents the pressure that remains in the lung after the completion of each breath. The volume of the lung above its rest volume at the end of each breath is denoted by $V_{\mathrm{ex}}$ and is related to $P_{\mathrm{ex}}$ through the equation $P_{\mathrm{ex}}=V_{\mathrm{ex}} / C\left(V_{\mathrm{ex}}\right)$. During inspiration, $P_{\mathrm{vent}}=$ $P_{\text {set }}$ and during expiration $P_{\text {vent }}=P_{\text {peep }}$. For the elastic pressures, we assume that $P_{\text {elastic }}=V(t) / C\left(V+V_{\mathrm{ex}}\right)$. Lastly, it is assumed that the resistive pressure is proportional to the flows in and out of the lung: $P_{\text {resistive }}=R V$. The volume in the lung (above $V_{\mathrm{ex}}$ ) is then given by the ordinary differential equations:

Inspiration

$$
\begin{aligned}
& R_{i}\left(\frac{\mathrm{d} V_{i}}{\mathrm{~d} t}\right)+\frac{V_{i}}{C_{i}\left(V_{i}+V_{\mathrm{ex}}\right)}+P_{\mathrm{ex}}=P_{\mathrm{set}}, \\
& 0 \leq t \leq t_{i}
\end{aligned}
$$

Expiration

$$
\begin{aligned}
& R_{e}\left(\frac{\mathrm{d} V_{e}}{\mathrm{~d} t}\right)+\frac{V_{e}}{C_{e}\left(V_{e}+V_{\mathrm{ex}}\right)}+P_{\mathrm{ex}}=P_{\mathrm{peep}}, \\
& t_{i} \leq t \leq t_{\mathrm{tot}} .
\end{aligned}
$$

It is assumed that the $V_{i}(0)=0$ and $V_{e}\left(t_{i}\right)=V_{i}\left(t_{i}\right)=V_{T}$ (the tidal volume) and serve as initial conditions for Eqs. (2.2) and (2.3), respectively. The unknown endexpiratory pressure $P_{\mathrm{ex}}$ and end-expiratory volume $V_{\mathrm{ex}}$ are determined by the equation $V_{e}\left(t_{\mathrm{tot}}\right)=0$. The initial-value problems for inspiratory and expiratory phases can be solved exactly. For example, one finds for the inspiratory volume:

$$
\begin{aligned}
V_{i}(t)= & \frac{a_{i}+b_{i} V_{\mathrm{ex}}}{b_{i}\left(1+b_{i}\left(P_{\mathrm{set}}-P_{\mathrm{ex}}\right)\right)}\left[b_{i}\left(P_{\mathrm{set}}-P_{\mathrm{ex}}\right)\right. \\
& \left.-\Phi\left(-b_{i}\left(P_{\mathrm{set}}-P_{\mathrm{ex}}\right) e^{\left.-b_{i}\left(P_{\mathrm{set}}-P_{\mathrm{ex}}\right)-\frac{\left(b_{i}\left(P_{\mathrm{set}}-P_{\mathrm{ex}}\right)-1\right)^{2} t}{\left(a_{i}+b_{i} \mathrm{ve}_{\mathrm{e}}\right) R_{i}}\right)}\right)\right]
\end{aligned}
$$

where the parameters $a_{i}$ and $b_{i}$ define the compliance functions: $C_{i}\left(V_{i}+V_{\mathrm{ex}}\right)=a_{i}+b_{i}\left(V_{i}+V_{\mathrm{ex}}\right)$. Here $\Phi$ is the productlog function i.e. $\Phi(z)$ is a branch of the solutions of $z=u e^{u}$. A similar expression can be computed for $V_{e}(t)$ which depends on the compliance function $C_{e}\left(V_{e}+V_{\mathrm{ex}}\right)=a_{e}+b_{e}\left(V_{e}+V_{\mathrm{ex}}\right)$. The endexpiratory pressure is determined by solving the equation $V_{e}\left(t_{\mathrm{tot}}\right)=0$ for $P_{\mathrm{ex}}$ and $V_{\mathrm{ex}}$, using the fact that
$P_{\mathrm{ex}}=V_{\mathrm{ex}} / C_{e}\left(V_{\mathrm{ex}}\right)$. Due to the transcendental nature of this equation, this equation must be solved numerically. Having the solution for the one-segment model, we extend it to a model where the inspiratory and expiratory parts of the breathing cycle are broken down into three segments upon which the compliance functions are of the above form.

\section{THE THREE-SEGMENT MODEL}

Svantesson et al. (1998) calculated the elastic pressurevolume $\left(P_{\text {elastic }}-V\right)$ curves of 15 healthy anesthetized adults in a study of lung recruitment as a means of improving ventilator management. Using specific and averaged values for the 15 subjects, they constructed the $P_{\text {elastic }}-V$ curves and found that the compliance of the lungs during inflation transverses three zones: increasing compliance, constant compliance, and decreasing compliance. During inflation from low lung volumes, the compliance increases linearly with volume up to a particular lung volume, which we denote by $V_{i_{1}}^{s}$, at which point the compliance remains constant up to a second particular volume, $V_{i_{2}}^{s}>V_{i_{1}}^{s}$, at which point the compliance decreases linearly. Depending on the applied pressures, $P_{\text {set }}$ and $P_{\text {peep }}$, the inflation cycle may encompass one, two, or all three of these segments. This type of compliance function is pictured in Fig. 1. This piecewise linear compliance function for inspiration can be represented mathematically as

$$
C_{i}\left(V_{i}\right)= \begin{cases}a_{i_{1}}+b_{i_{1}} V_{i} & \text { if } 0 \leq V_{i} \leq V_{i_{1}}^{s} \\ a_{i_{2}} & \text { if } V_{i_{1}}^{s} \leq V_{i} \leq V_{i_{2}}^{s} \\ a_{i_{3}}+b_{i_{3}} V_{i} & \text { if } V_{i_{2}}^{s} \leq V_{i} \leq V_{T} .\end{cases}
$$

The $P_{\text {elastic }}-V$ curve for this type of compliance function is pictured in Fig. 2. Similar types of figures can be drawn for the expiratory compliance function $C_{e}(V)$ :

$$
C_{e}\left(V_{e}\right)= \begin{cases}a_{e_{3}}+b_{e_{3}} V_{e} & \text { if } 0 \leq V_{e} \leq V_{e_{2}}^{s} \\ a_{e_{2}} & \text { if } V_{e_{2}}^{s} \leq V_{e} \leq V_{e_{1}}^{s} \\ a_{e_{1}}+b_{e_{1}} V_{e} & \text { if } V_{e_{1}}^{s} \leq V_{e} \leq V_{T} .\end{cases}
$$

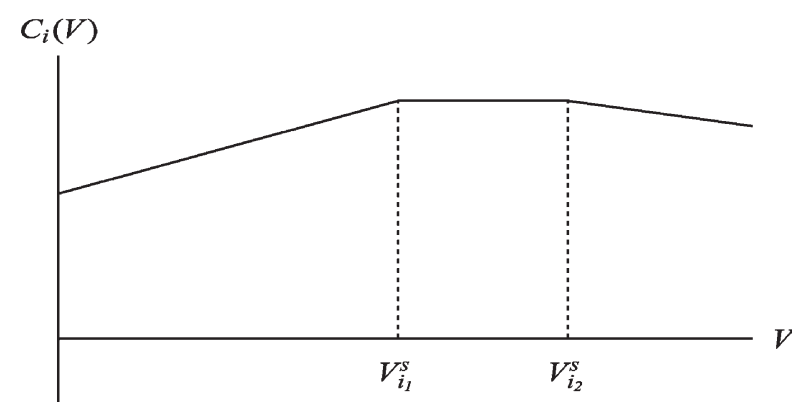

FIGURE 1 Three-segment compliance model: lung inspiratory compliance versus lung volume. 
The basic one-segment model (Eqs. (2.2) and (2.3)) can now be generalized to the three-segment model by assuming that during inspiration and expiration, the compliance functions, $C_{i}(V)$ and $C_{e}(V)$ are changing according to Eqs. (3.1) and (3.2). In particular, we assume that the lung volumes satisfy the following system of differential equations:

Inspiration

$$
\begin{aligned}
& R_{i}\left(\frac{\mathrm{d} V_{i_{1}}}{\mathrm{~d} t}\right)+\frac{V_{i_{1}}}{a_{i_{1}}+b_{i_{1}}\left(V_{i_{1}}+V_{\mathrm{ex}}\right)}+P_{\mathrm{ex}}=P_{\mathrm{set}}, \\
& 0 \leq t \leq t_{i_{1}} \\
& R_{i}\left(\frac{\mathrm{d} V_{i_{2}}}{\mathrm{~d} t}\right)+\frac{V_{i_{2}}}{a_{i_{2}}}+P_{\mathrm{ex}}=P_{\mathrm{set}}, \quad t_{i_{1}} \leq t \leq t_{i_{2}} \\
& R_{i}\left(\frac{\mathrm{d} V_{i_{3}}}{\mathrm{~d} t}\right)+\frac{V_{i_{3}}}{a_{i_{3}}+b_{i_{3}}\left(V_{i_{3}}+V_{\mathrm{ex}}\right)}+P_{\mathrm{ex}}=P_{\mathrm{set}}, \\
& t_{i_{2}} \leq t \leq t_{i}
\end{aligned}
$$

\section{Expiration}

$$
\begin{aligned}
& R_{e}\left(\frac{\mathrm{d} V_{e_{1}}}{\mathrm{~d} t}\right)+\frac{V_{e_{1}}}{a_{e_{1}}+b_{e_{1}}\left(V_{e_{1}}+V_{\mathrm{ex}}\right)}+P_{\mathrm{ex}}=P_{\mathrm{peep}}, \\
& t_{i} \leq t \leq t_{e_{1}} \\
& R_{e}\left(\frac{\mathrm{d} V_{e_{2}}}{\mathrm{~d} t}\right)+\frac{V_{e_{2}}}{a_{e_{2}}}+P_{\mathrm{ex}}=P_{\mathrm{peep}}, \quad t_{e_{1}} \leq t \leq t_{e_{2}} \\
& R_{e}\left(\frac{\mathrm{d} V_{e_{3}}}{\mathrm{~d} t}\right)+\frac{V_{e_{3}}}{a_{e_{3}}+b_{e_{3}}\left(V_{e_{3}}+V_{\mathrm{ex}}\right)}+P_{\mathrm{ex}}=P_{\mathrm{peep}}, \\
& t_{e_{2}} \leq t \leq t_{\mathrm{tot}} .
\end{aligned}
$$

In Eqs. (3.5)-(3.8), we require that the solutions satisfy the continuity conditions: $V_{i_{1}}\left(t_{i_{1}}\right)=V_{i_{2}}\left(t_{i_{1}}\right), V_{i_{2}}\left(t_{i_{2}}\right)=V_{i_{3}}\left(t_{i_{2}}\right)$, $V_{i_{3}}\left(t_{i}\right)=V_{e_{1}}\left(t_{i}\right), \quad V_{e_{1}}\left(t_{e_{1}}\right)=V_{e_{2}}\left(t_{e_{1}}\right), \quad$ and $\quad V_{e_{2}}\left(t_{e_{2}}\right)=$ $V_{e_{3}}\left(t_{e_{2}}\right)$. We also have the initial and boundary conditions: $V_{i_{1}}(0)=0, V_{i_{3}}\left(t_{i}\right)=V_{T}$, and $V_{e_{3}}\left(t_{\text {tot }}\right)=0$. These continuity conditions require the following restrictions be placed on the parameters of the compliance functions:

$$
\begin{gathered}
a_{i_{2}}=a_{i_{1}}+b_{i_{1}} V_{i_{1}}^{s} \\
a_{i_{3}}=a_{i_{2}}-b_{i_{3}} V_{i_{2}}^{s} \\
a_{e_{2}}=a_{e_{1}}+b_{e_{1}} V_{e_{1}}^{s} \\
a_{e_{3}}=a_{e_{2}}-b_{e_{3}} V_{e_{2}}^{s} .
\end{gathered}
$$

As in the case of the basic one-segment model, the endexpiratory pressure is determined by numerically solving for $P_{\text {ex }}$ the equation: $V_{e_{3}}\left(t_{\text {tot }}\right)=0$.

The parameters, $V_{i_{1}}^{s}$ and $V_{i_{2}}^{s}$ for inspiration and $V_{e_{1}}^{s}$ and $V_{e_{2}}^{s}$ for expiration, are inputs for the model. The times, $t_{i_{1}}, t_{i_{2}}, t_{e_{1}}, t_{e_{2}}$, that appear in Eqs. (3.5)-(3.8) are determined by the equations, $V_{i_{1}}\left(t_{i_{1}}\right)=V_{i_{1}}^{s}, V_{i_{2}}\left(t_{i_{2}}\right)=$ $V_{i_{2}}^{s}, \quad V_{e_{1}}\left(t_{e_{1}}\right)=V_{e_{1}}^{s}, \quad$ and $V_{e_{2}}\left(t_{e_{2}}\right)=V_{e_{2}}^{s}$, respectively.
These equations can be solved analytically for the transition times:

$$
\begin{aligned}
t_{i_{1}}= & -\frac{R_{i}}{\left(1-b_{i_{1}}\left(P_{\mathrm{set}}-P_{\mathrm{ex}}\right)\right)^{2}} \\
& \times\left[b_{i_{1}}\left(1-b_{i_{1}}\left(P_{\mathrm{set}}-P_{\mathrm{ex}}\right)\right) V_{i_{1}}^{s}+\left(a_{i_{1}}+b_{i} V_{\mathrm{ex}}\right) \log \Psi\right]
\end{aligned}
$$

$$
\begin{aligned}
t_{i_{2}}= & t_{i_{1}}-\left(a_{i_{1}}+b_{i_{1}} V_{\mathrm{ex}}+b_{i_{1}} V_{i_{1}}^{s}\right) R_{i} \\
& \times \log \left(\frac{\left(a_{i_{1}}+b_{i_{1}} V_{\mathrm{ex}}+b_{i_{1}} V_{i_{1}}^{s}\right)\left(P_{\mathrm{set}}-P_{\mathrm{ex}}\right)-V_{i_{2}}}{\left(a_{i_{1}}+b_{i_{1}} V_{\mathrm{ex}}+b_{i_{1}} V_{i_{1}}^{s}\right)\left(P_{\mathrm{set}}-P_{\mathrm{ex}}\right)-V_{i_{1}}}\right)
\end{aligned}
$$

where

$$
\Psi=\frac{\left(a_{i_{1}}+b_{i} V_{\mathrm{ex}}+b_{i_{1}} V_{i_{1}}^{s}\right)\left(P_{\mathrm{set}}-P_{\mathrm{ex}}\right)-V_{i_{1}}^{s}}{\left(a_{i_{1}}+b_{i} V_{\mathrm{ex}}\right)\left(P_{\mathrm{set}}-P_{\mathrm{ex}}\right)}
$$

and

$$
\begin{aligned}
t_{e_{1}}= & -\frac{R_{e}}{\left(1+b_{e_{1}} P_{\mathrm{ex}}\right)^{2}}\left[b_{e_{1}}\left(1+b_{i_{1}} P_{\mathrm{ex}}\left(V_{i_{1}}^{s}-V_{T}\right)\right)\right. \\
+ & \left.\left(a_{e_{1}}+b_{e_{1}} V_{\mathrm{ex}}\right) \log \Phi\right] \\
t_{e_{2}}= & t_{e_{1}}-R_{e}\left(a_{e_{1}}+b_{e_{1}} V_{\mathrm{ex}}+b_{e_{1}} V_{e_{1}}^{s}\right) \\
& \times \log \left(\frac{V_{e_{2}}^{s}+a_{e_{1}}+b_{e_{1}}\left(V_{e_{1}}^{s}+V_{\mathrm{ex}}\right)}{V_{e_{1}}^{s}+a_{e_{1}}+b_{e_{1}}\left(V_{e_{1}}^{s}+V_{\mathrm{ex}}\right)}\right)
\end{aligned}
$$

where

$$
\Phi=\frac{a_{e_{1}}+b_{e_{1}}\left(V_{e_{1}}^{s}+V_{\mathrm{ex}}\right) P_{\mathrm{ex}}+V_{e_{1}}^{s}}{a_{e_{1}}+b_{e_{1}}\left(V_{T}+V_{\mathrm{ex}}\right) P_{\mathrm{ex}}+V_{T}}
$$

In Fig. 3, the three-segment, variable compliance model is used to calculate the lung volume over one cycle for parameter settings that are given in the caption of the figure. The $P_{\text {elastic }}-V$ curve for this simulation is shown in Fig. 4 and graphs of the compliance functions are shown in Fig. 5. The vertical lines in this figure indicate the

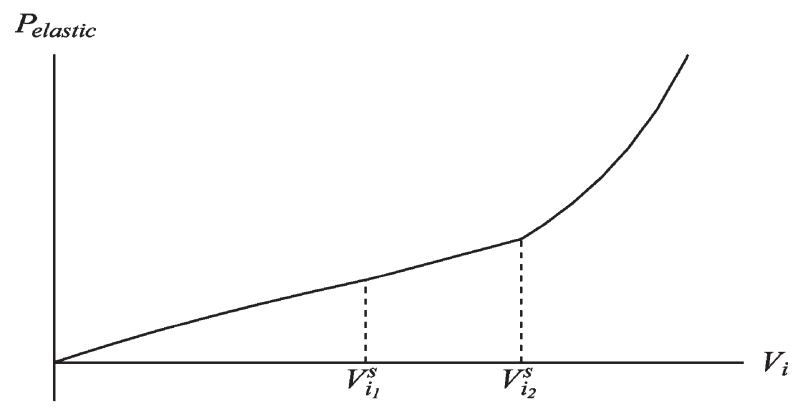

FIGURE $2 P_{\text {elastic }}-V$ curve inspiration for the three-segment model. 


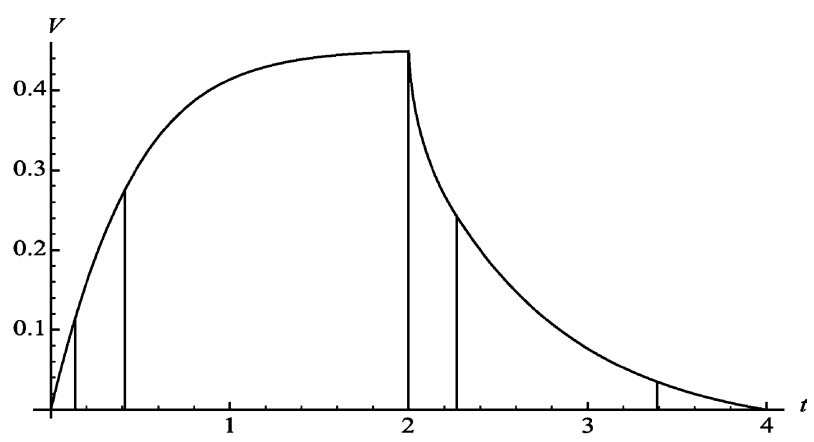

FIGURE 3 Lung volume curve over one breath for three-segment model $V_{i_{1}}^{s}=0.32 \mathrm{~L}, V_{i_{2}}^{s}=0.48 \mathrm{~L}, V_{e_{1}}^{s}=0.44 \mathrm{~L}, V_{e_{2}}^{s}=0.24, a_{i_{1}}=$ $0.016 \mathrm{~L} / \mathrm{cm} \mathrm{H} \mathrm{H}_{2} \mathrm{O}, a_{i_{2}}=0.026 \mathrm{~L} / \mathrm{cm} \mathrm{H}_{2} \mathrm{O}, a_{i_{3}}=0.0 .034 \mathrm{~L} / \mathrm{cm} \mathrm{H}_{2} \mathrm{O}$, $b_{i_{1}}=0.0321 / \mathrm{cm} \mathrm{H}_{2} \mathrm{O}, b_{i_{3}}=-0.0161 / \mathrm{cm} \mathrm{H}_{2}, a_{e_{1}}=0.113 \mathrm{~L} / \mathrm{cm} \mathrm{H}_{2} \mathrm{O}$, $a_{e_{2}}=0.039 \mathrm{~L} / \mathrm{cm} \mathrm{H} \mathrm{H}_{2} \mathrm{O}, a_{e_{3}}=0.019 \mathrm{~L} / \mathrm{cm} \mathrm{H} \mathrm{H}_{2} \mathrm{O}, b_{e_{1}}=-0.1661 / \mathrm{cm}$ $\mathrm{H}_{2}, b_{e_{3}}=0.0821 / \mathrm{cm} \mathrm{H}_{2}, R_{i}=R_{e}=20 \mathrm{~cm} \mathrm{H}_{2} \mathrm{O} / \mathrm{L} / \mathrm{s}, P_{\text {set }}=25 \mathrm{~cm} \mathrm{H}_{2} \mathrm{O}$, $P_{\text {peep }}=5 \mathrm{~cm} \mathrm{H}_{2} \mathrm{O}, t_{i}=2 \mathrm{~s}$, and $t_{\text {tot }}=4 \mathrm{~s}$. The vertical lines denote the times at which the form of the compliance function is changing.

times over the breathing cycle at which there is a change in the compliance function, i.e. $t_{i_{1}}, t_{i_{2}}, t_{i}, t_{i}+t_{e_{1}}$ and $t_{i}+$ $t_{e_{2}}$. In Fig. $3, P_{\mathrm{ex}}=5.7391 \mathrm{~cm} \mathrm{H}_{2} \mathrm{O}, V_{\mathrm{ex}}=0.205 \mathrm{~L}, t_{i_{1}}=$ $0.136 \mathrm{~s}, t_{i_{2}}=0.413 \mathrm{~s}, t_{i}+t_{e_{1}}=2.268 \mathrm{~s}$ and $t_{i}+t_{e_{2}}=$ $3.390 \mathrm{~s}$. The model does exhibit hysteresis in the $P_{\text {elastic }}-V$ curve and for this example the compliance functions for inspiration and expiration are different. In Fig. 4, the arrows pointing upward are during inspiration and downward during expiration. The choices for parameters $\left(V_{i_{k}}, V_{e_{k}}, a_{i_{m}}, b_{i_{n}}, a_{e_{m}}, b_{e_{n}}\right)$ for this simulation were taken to illustrate the generality of the model. Values for these parameters that come from human data will be used in the next section of the paper.

It is worthwhile to see the effect of the variable compliance on the lung volume. In Fig. 6, the lung volumes are graphed for the variable compliance model (solid curve) and the constant compliance model (dashed curve). For $C$ in the constant compliance model, we have used the average compliance for the three-segment model during inspiration and expiration $\left(C_{\text {ave }}\right)$. The average

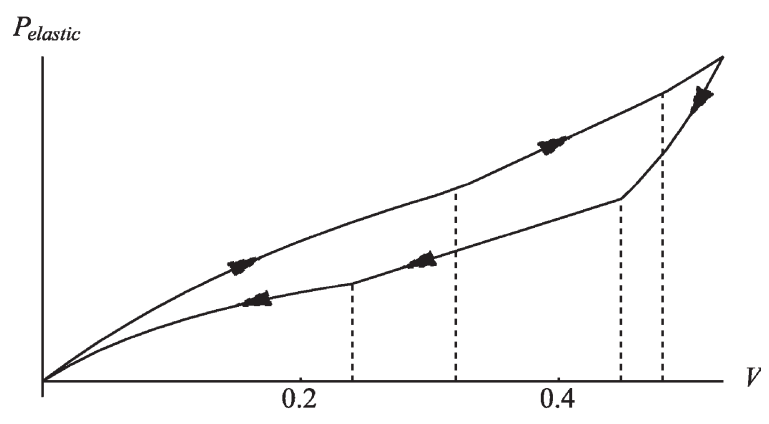

FIGURE $4 P_{\text {elastic }}-V$ curve used in simulation for Fig. 3. The vertical dashed lines denote the volumes at which the form of the compliance function is changing form. The arrowheads indicate inspiration or expiration. compliance was calculated using the formula:

$$
\begin{aligned}
C_{\mathrm{ave}}= & \left(\frac{D}{V_{T}}\right) \sum_{j=1}^{3}\left(\int_{V_{i_{j-1}}+V_{\mathrm{ex}}}^{V_{i_{j}}+V_{\mathrm{ex}}} C_{i}(V) \mathrm{d} V\right) \\
& +\left(\frac{1-D}{V_{T}}\right) \sum_{j=1}^{3}\left(\int_{V_{e_{j-1}}+V_{\mathrm{ex}}}^{V_{e_{j}}+V_{\mathrm{ex}}} C_{e}(V) \mathrm{d} V\right)
\end{aligned}
$$

where $V_{i_{0}}=V_{e_{3}}=0$ and $V_{i_{3}}=V_{e_{0}}=V_{T}$. We note the differences in the two models, in particular, the higher lung volume for the constant compliance model. However, this is not the general rule and by changing the compliance function parameters it is possible to have the tidal volume for the variable compliance model larger than the constant compliance model.

Important linkages exist between clinician set parameters: applied airway pressure $\left(P_{\text {set }}\right.$ and $\left.P_{\text {peep }}\right)$, frequency of breathing $(f)$, inspiratory time fraction $\left(D=t_{i} / t_{\text {tot }}\right)$ and clinically important outcomes: end-expiratory pressure $\left(P_{\mathrm{ex}}\right)$, tidal volume $\left(V_{T}\right)$, mean alveolar pressure $\left(P_{m}\right)$, and average lung volume $\left(V_{\text {ave }}\right)$. The mean alveolar pressure is defined as the average elastic pressure over one breath plus the end-expiratory pressure $P_{\mathrm{ex}}$. In particular, it is defined mathematically as

$$
\begin{aligned}
P_{m}= & \left(\frac{D}{t_{i}}\right) \sum_{j=1}^{3} \int_{t_{i_{j-1}}}^{t_{i j}} \frac{V_{i_{j}}(t)}{C_{i_{j}}\left(V_{i_{j}}(t)+V_{\mathrm{ex}}\right)} \mathrm{d} t \\
& +\left(\frac{1-D}{t_{\mathrm{tot}}-t_{i}}\right) \sum_{j=1}^{3} \int_{t_{e_{j-1}}}^{t_{e_{j}}} \frac{V_{e_{j}}(t)}{C_{e_{j}}\left(V_{e_{j}}(t)+V_{\mathrm{ex}}\right)} \mathrm{d} t+P_{\mathrm{ex}}
\end{aligned}
$$

The intervals, $\left[t_{i_{j-1}}, t_{i_{j}}\right]$ and $\left[t_{e_{j-1}}, t_{e_{j}}\right]$, are the subintervals of $\left[0, t_{i}\right]$ and $\left[t_{i}, t_{\text {tot }}\right]$, respectively, for the different segments in the variable compliance model. The average lung volume is defined in an analogous way. The linkage between the key outcome variables and the frequency has been studied in the constant compliance case by Marini et al. (Marini et al., 1989; Marini and Crooke, 1993). It was shown in these papers that in different types of lung diseases (e.g. obstructive and restrictive), the mathematical model gave robust predictions for the key outcome variables and theoretically provided the clinician with a means of choosing the input parameters of mechanical ventilation that optimize the desired results. In keeping with this spirit, we offer predictions of the three-segment model for the key outcome variables as functions of $f$, using clinically observable values for the applied pressures and inspiratory time fraction. In particular, we compare these with the predictions of the constant compliance model (using the average compliance of the three-segment model) and probe for meaningful differences. In the simulations for Fig. 7, the following values for the physiologic and ventilator parameters have been chosen: $R_{i}=R_{e}=20 \mathrm{~cm} \mathrm{H} \mathrm{H}_{2} \mathrm{O} / \mathrm{l} / \mathrm{s}, P_{\text {set }}=25 \mathrm{~cm} \mathrm{H} \mathrm{H}_{2} \mathrm{O}$, $P_{\text {peep }}=5 \mathrm{~cm} \mathrm{H}_{2} \mathrm{O}$, and $D=0.5$. The values of the parameters in the compliance functions are in Fig. 3. 

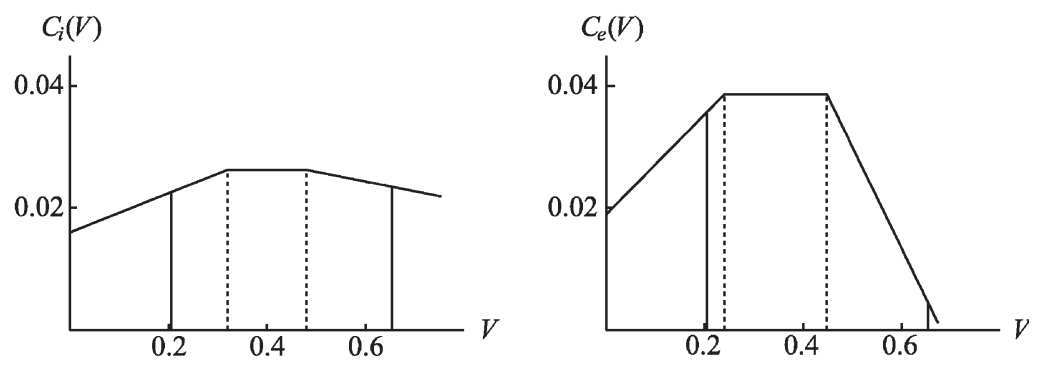

FIGURE 5 Compliance functions used in simulation for Fig. 3. The dashed lines are the volumes at which the compliance functions are changing form and the solid lines are $V_{\mathrm{ex}}$ and $V_{T}+V_{\mathrm{ex}}$.

In these simulations, there is hysteresis in the $P_{\text {elastic }}-V$ curves. The compliances in the constant compliance model simulations are the average compliance of the three-segment models and range from $C_{\text {ave }}=0.02671$ to $0.02841 / \mathrm{cm} \mathrm{H}_{2} \mathrm{O}$. For $f=5-18$, all three segments of the inspiratory and expiratory compliance functions are tranversed. For $f=19-35$, three segments are used during inspiration and two segments during expiration. For $f=36-40$, two segments of the inspiratory and expiratory compliance functions are traversed. In this last set of simulations, $V_{\text {ex }}>V_{i_{1}}^{s}, V_{e_{2}}^{s}$. We note some differences between the constant compliance and threesegment models for clinically relevant values of the physiologic and ventilator parameters. In particular, there are differences in the tidal volume and average volumes at the lower frequencies and the mean alveolar pressures at the high frequencies. The differences in the end-expiratory pressures are insignificant. The greatest percentage difference in tidal volumes was $16.5 \%$ at $f=8$. The greatest difference in average volumes was $13.5 \%$ and $13.6 \%$ in mean alveolar pressures. A similar comparison can be done for the key outcome variables versus the inspiratory time fraction $D$.

\section{RECRUITMENT AND THE THREE-SEGMENT MODEL}

The value of any mathematical model for a physiologic process lies in its utility to predict the outcome of

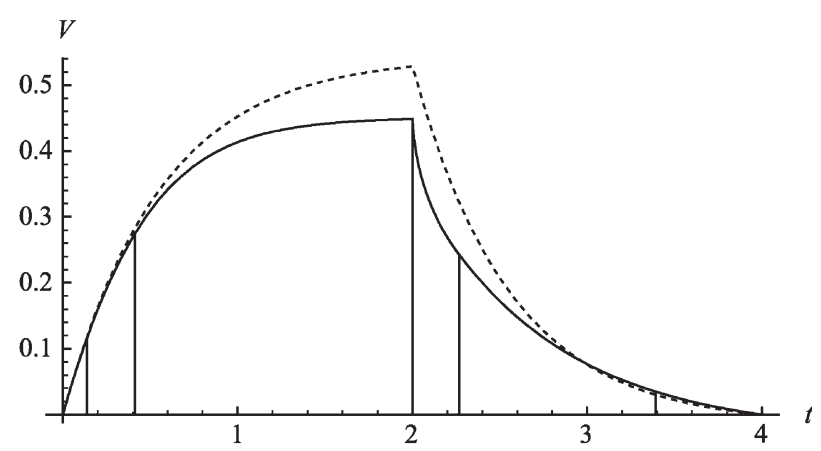

FIGURE 6 Constant compliance and three-segment models: The solid curve is the lung volume of the three-segment model in Fig. 3 and the dashed curve is the volume curve for the constant compliance model with $C=C_{\text {ave }}=0.107$. the process in a realistic setting. In this spirit we use the variable compliance model to predict key clinical outcomes (tidal volume, average volume, mean alveolar pressure) using ranges of the input variables (frequency, applied airway pressure, duty cycle) that are found in the ICU and patient parameters (compliance and resistance) that characteristic of healthy or sick individuals. Experimental studies indicate that mechanical ventilation may lead to up-regulation of inflammatory mediators and exacerbate pulmonary injury. Two important factors in ventilator-associated lung injury (VALI) are alveolar
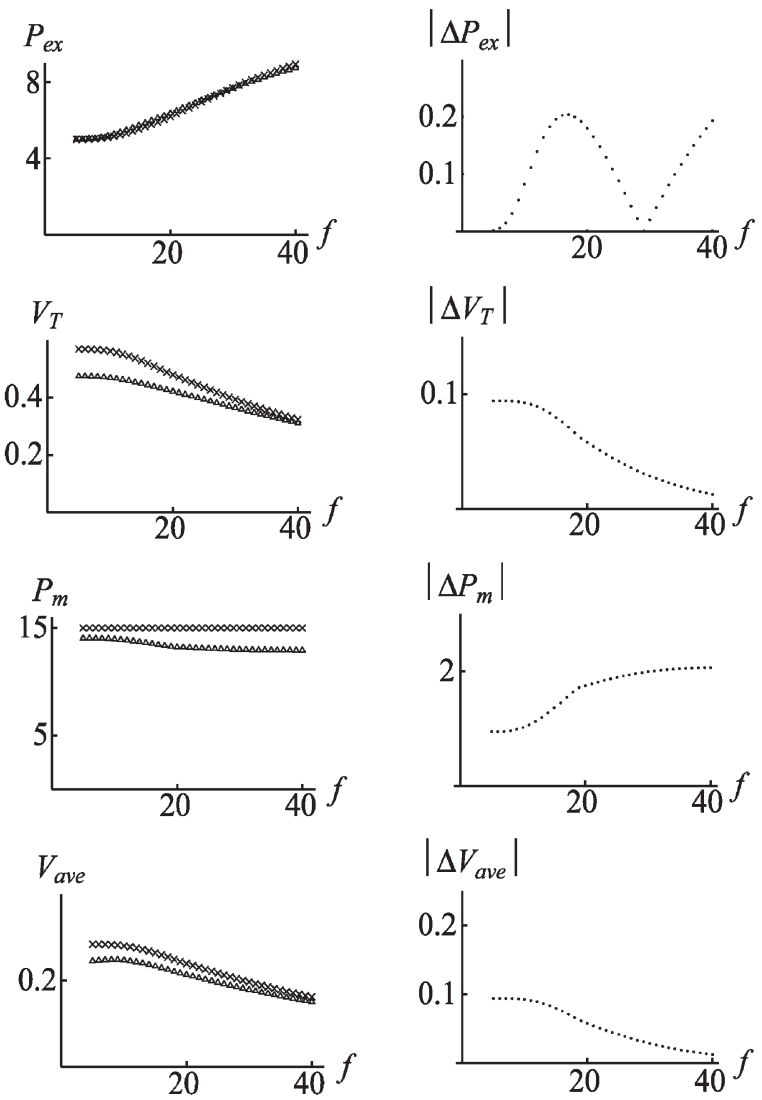

FIGURE 7 End-expiratory pressures, tidal volumes, mean alveolar pressures, and average volumes for constant and three-segment models as function of frequency (left panel) and their absolute differences (right panel). In the left panel graph, the triangles are quantities for the threesegment model and the crosses are for the constant compliance model. 
overdistension due to high transpulmonary pressures and repeated alveolar collapse and reopening due to ventilation at low lung volumes (Mancini et al., 2001). To keep alveolar extension within safe limits, it is suggested that tidal volumes be limited to $6-7 \mathrm{ml} / \mathrm{kg}$, applied inspiratory pressures less than $35 \mathrm{~cm} \mathrm{H}_{2} \mathrm{O}$, and applied PEEPs between $8-11 \mathrm{~cm} \mathrm{H}_{2} \mathrm{O}$. A dynamic model that predicts volumes and pressure during ventilation may aid in the implementation of such lung protective strategies.

The elastic pressure-volume relationship of the respiratory system during inspiration or expiration can be modeled using the three-segment model described in the previous section. During inspiration, the lower inflection point $\left(V_{i_{1}}^{s}\right)$ of the elastic pressure-volume curve can be used to establish a theoretical optimal value for PEEP in patients with acute lung injury (ALI) and/or acute respiratory distress syndrome (ARDS) (Maggiore et al., 2001). It has been demonstrated (Amato et al., 1998; Papadakos and Lachmann, 2002) that a substantial reduction of mortality in ARDS patients was achieved by using tidal volumes and applied PEEPs that result in inflation volumes that are between the two inflection points of the static elastic pressure-volume curve.

Svantesson et al. (1998) studied 15 healthy adults (9 females and 6 males) to determine the effect of recruitment maneuvers on their $P_{\text {elastic }}-V$ curves. The recruitment maneuver involved inflating the lungs to a pressure of $40 \mathrm{~cm} \mathrm{H}_{2} \mathrm{O}$ for a period of $15 \mathrm{~s}$. They measured variables that can be used to calculate the parameters in the three-segment model (assuming no hysteresis in the $P_{\text {elastic }}-V$ curves) before and after the recruitment maneuver. Their data has been used to calculate $V_{i_{1}}^{s}, V_{i_{2}}^{s}$, $a_{i_{1}}, b_{i_{1}}, a_{i_{3}}$, and $b_{i_{3}}$ for each patient. A summary of the calculations is presented in Tables I and II. We note that data from patient 9 is not listed because of missing data in the original work (Svantesson et al., 1998). Although averages might be deemed of little value on a small data set, it is worth noting that the upper and lower inflection

TABLE I Compliance function parameters for patients before recruitment maneuver

\begin{tabular}{lcccccc}
\hline Patient & $V_{i_{1}}^{s}$ & $V_{i_{2}}^{s}$ & $a_{i_{1}}$ & $b_{i_{1}}$ & $a_{i_{3}}$ & $b_{i_{3}}$ \\
\hline 1 & 1.069 & 1.66 & 0.0521 & 0.0223 & 0.102 & -0.0156 \\
2 & 1.927 & 2.44 & 0.0405 & 0.0392 & 0.188 & -0.0295 \\
3 & 0.800 & 2.37 & 0.0364 & 0.0632 & 0.129 & -0.0179 \\
4 & 1.230 & 2.06 & 0.0504 & 0.0510 & 0.146 & -0.0156 \\
5 & 2.217 & 2.77 & 0.0685 & 0.0377 & 0.248 & -0.0348 \\
6 & 1.016 & 2.75 & 0.0353 & 0.0677 & 0.159 & -0.0201 \\
7 & 3.074 & 3.48 & 0.0500 & 0.0421 & 0.274 & -0.0271 \\
8 & 2.542 & 3.61 & 0.0624 & 0.0356 & 0.313 & -0.0444 \\
10 & 3.176 & 3.57 & 0.0513 & 0.0594 & 0.370 & -0.0378 \\
11 & 2.124 & 3.89 & 0.0457 & 0.0783 & 0.352 & -0.0359 \\
12 & 1.343 & 3.26 & 0.0419 & 0.0671 & 0.257 & -0.0382 \\
13 & 1.814 & 3.07 & 0.0518 & 0.0448 & 0.267 & -0.0437 \\
14 & 1.760 & 4.07 & 0.0604 & 0.0498 & 0.246 & -0.0240 \\
15 & 1.866 & 2.28 & 0.0272 & 0.0765 & 0.248 & -0.0344 \\
Average & 1.854 & 2.949 & 0.0527 & 0.0492 & 0.228 & -0.0286 \\
\hline
\end{tabular}

Calculations based upon data taken from Svantesson et al. (1998). points, on average, are lowered by the recruitment maneuvers on these healthy individuals.

Using the values of the parameters in Tables I and II, simulations were performed to compute lung volume profiles and key outcome variables, before and after recruitment, using pressure controlled ventilation. For these simulations, the following ventilation parameters were chosen: $P_{\text {set }}=30 \mathrm{~cm} \mathrm{H}_{2} \mathrm{O}, P_{\text {peep }}=5 \mathrm{~cm} \mathrm{H}_{2} \mathrm{O}, t_{\text {tot }}=$ $3 \mathrm{~s}, D=0.5, R_{i}=3 \mathrm{~cm} \mathrm{H}{ }_{2} \mathrm{O} / 1 / \mathrm{s}$, and $R_{e}=5 \mathrm{~cm} \mathrm{H}_{2} \mathrm{O} / 1 / \mathrm{s}$. Simulations for patient 1 are shown in Fig. 8. The values for the key outcome variables are: (before) $V_{T}=1.730$, $V_{\mathrm{ave}}=0.953, V_{\mathrm{ex}}=0.313, P_{\mathrm{ex}}=5.292, P_{m}=18.155$, and $C_{\text {ave }}=0.072$; (after) $V_{T}=1.508, \quad V_{\text {ave }}=0.877$, $V_{\mathrm{ex}}=0.494, P_{\mathrm{ex}}=5.644, P_{m}=15.235$, and $C_{\mathrm{ave}}=$ 0.088. In this case, the tidal volume, average volume, and mean alveolar pressure decrease after recruitment. One should notice that the flat part of the compliance function has been extended considerably and the inflection points, $V_{i_{1}}^{s}$ and $V_{i_{2}}^{s}$, have been lowered.

In Fig. 9, the lung volumes and compliance functions for patient 2 are shown before and after the recruitment maneuver. The values for the key outcome variables are: (before) $V_{T}=2.534, \quad V_{\text {ave }}=1.393, \quad V_{\text {ex }}=0.298$, $P_{\mathrm{ex}}=5.7085, P_{m}=19.141$, and $C_{\mathrm{ave}}=0.095$; (after) $V_{T}=1.925, \quad V_{\text {ave }}=1.095, \quad V_{\mathrm{ex}}=0.556, P_{\mathrm{ex}}=5.900$, $P_{m}=16.991$, and $C_{\text {ave }}=0.093$. In this individual, we observe a similar change in the key outcome variables as was seen in patient 1 . However, there is one important difference for patient 2 after the recruitment maneuver: the ventilation starts above the lower inflection point. Looking at the compliance functions (before and after), we see that the plateau region of the compliance function (the region that is often targeted by clinicians) is increased.

One might ask the question: What is the effect of the recruitment maneuvers on the parameters in the compliance functions for the 14 patients? One approach is to analyze changes in the parameters that make up the compliance functions for inspiration or expiration. On average, during inspiration, recruitment maneuvers
TABLE II Compliance function parameters for patients after recruitment maneuver

\begin{tabular}{lcccccc}
\hline Patient & $V_{i_{1}}^{s}$ & $V_{i_{2}}^{s}$ & $a_{i_{1}}$ & $b_{i_{1}}$ & $a_{i_{3}}$ & $b_{i_{3}}$ \\
\hline 1 & 0.575 & 1.38 & 0.0415 & 0.0930 & 0.169 & -0.0536 \\
2 & 0.421 & 1.97 & 0.0413 & 0.1300 & 0.157 & -0.0308 \\
3 & 0.990 & 1.53 & 0.0432 & 0.0573 & 0.160 & -0.0394 \\
4 & 1.308 & 1.93 & 0.0587 & 0.0545 & 0.172 & -0.0220 \\
5 & 0.801 & 2.22 & 0.0872 & 0.0784 & 0.218 & -0.0305 \\
6 & 1.167 & 1.68 & 0.0489 & 0.0627 & 0.155 & -0.0195 \\
7 & 1.148 & 3.03 & 0.0833 & 0.0903 & 0.361 & -0.0574 \\
8 & 1.984 & 2.16 & 0.0896 & 0.0345 & 0.253 & -0.0440 \\
10 & 1.805 & 2.79 & 0.1020 & 0.0504 & 0.320 & -0.0453 \\
11 & 2.531 & 2.54 & 0.1240 & 0.0339 & 0.283 & -0.0289 \\
12 & 1.392 & 2.00 & 0.0750 & 0.0618 & 0.252 & -0.0453 \\
13 & 0.661 & 1.79 & 0.0714 & 0.0871 & 0.233 & -0.0581 \\
14 & 1.513 & 2.88 & 0.0935 & 0.0419 & 0.309 & -0.0526 \\
15 & 0.514 & 1.19 & 0.0576 & 0.1190 & 0.148 & -0.0248 \\
Average & 1.200 & 2.078 & 0.0750 & 0.0528 & 0.217 & -0.0355 \\
\hline
\end{tabular}

Calculations based upon data taken from Svantesson et al. (1998). 

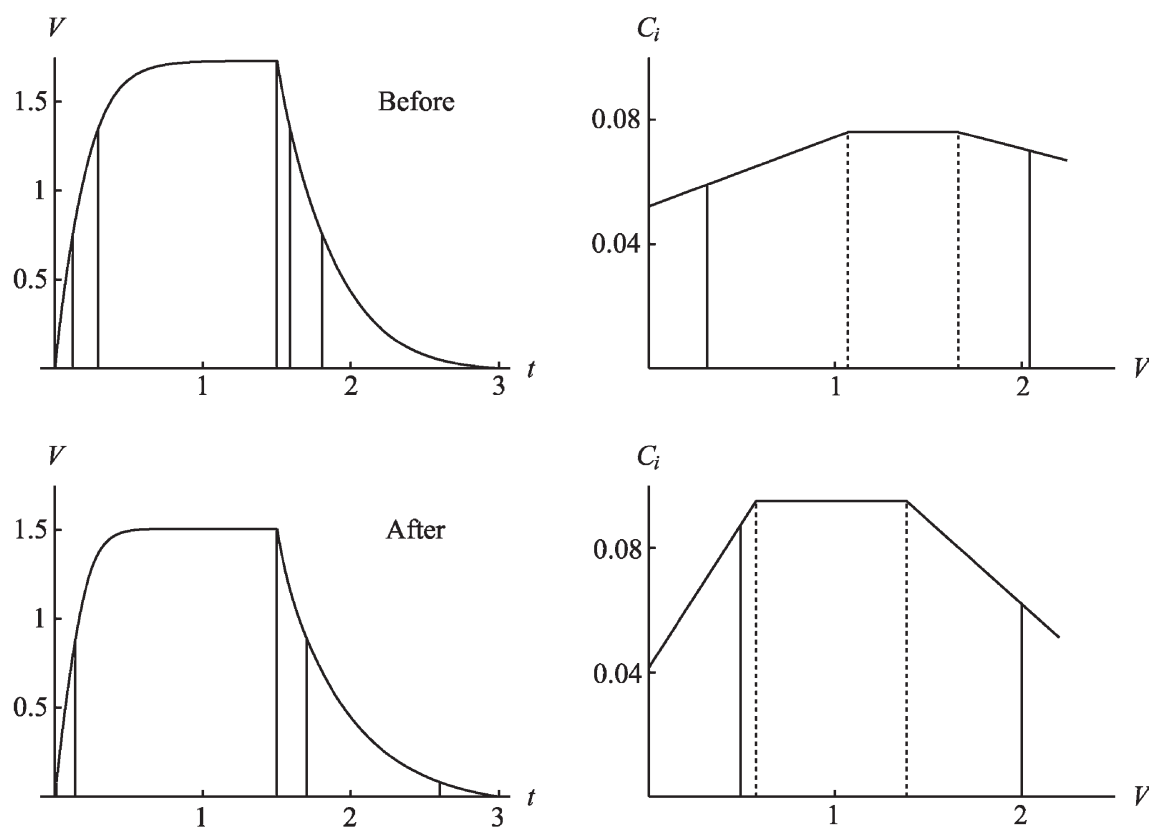

FIGURE 8 Volume and compliance functions before and after recruitment maneuver for patient 1 . In the graphs of the compliance functions, the dashed lines are volumes $V_{i_{1}}^{s}$ and $V_{i_{2}}^{s}$ and the solid vertical lines are $V_{\mathrm{ex}}$ and $V_{T}+V_{\mathrm{ex}}$.

decrease the lower inflection point volume, $V_{i_{2}}$, and increases the starting value of the compliance, $a_{i_{1}}$. The slopes of the initial segment, $b_{i_{1}}$ increase and on average, the slopes of the third segment, $b_{i_{3}}$, become less negative. However, there is variation in the other parameters from patient to patient. This may be indicative of the variability that exists among individuals with and without lung disease. A second measure of change is the effect of the recruitment maneuvers on the key outcome variables: $P_{\mathrm{ex}}$, $V_{T}, P_{m}$ and $V_{\text {ave }}$. In Table III, the tidal volumes, endexpiratory pressures, mean alveolar pressures, and average lung volume are computed for the 14 patients. From this table, we see that the tidal volume, average volumes, and mean alveolar pressures are lower by recruitment and there is little change in the end-expiratory pressure.

Variation in the applied PEEP for a fixed $P_{\text {set }}$ has some effect on the key outcome variables, $V_{T}, V_{\mathrm{ave}}, P_{\mathrm{ex}}$, and $P_{m}$, before and after recruitment. In Table IV, the tidal volumes, end-expiratory pressures, mean alveolar pressures, and average lung volumes, before and after recruitment, are shown for different levels of applied PEEP and $P_{\text {set }}=30$, using the compliance parameters for patient 1. For this particular situation, we see that tidal and average volumes decrease with increasing $P_{\text {peep }}$ while end-expiratory pressure and mean alveolar pressure
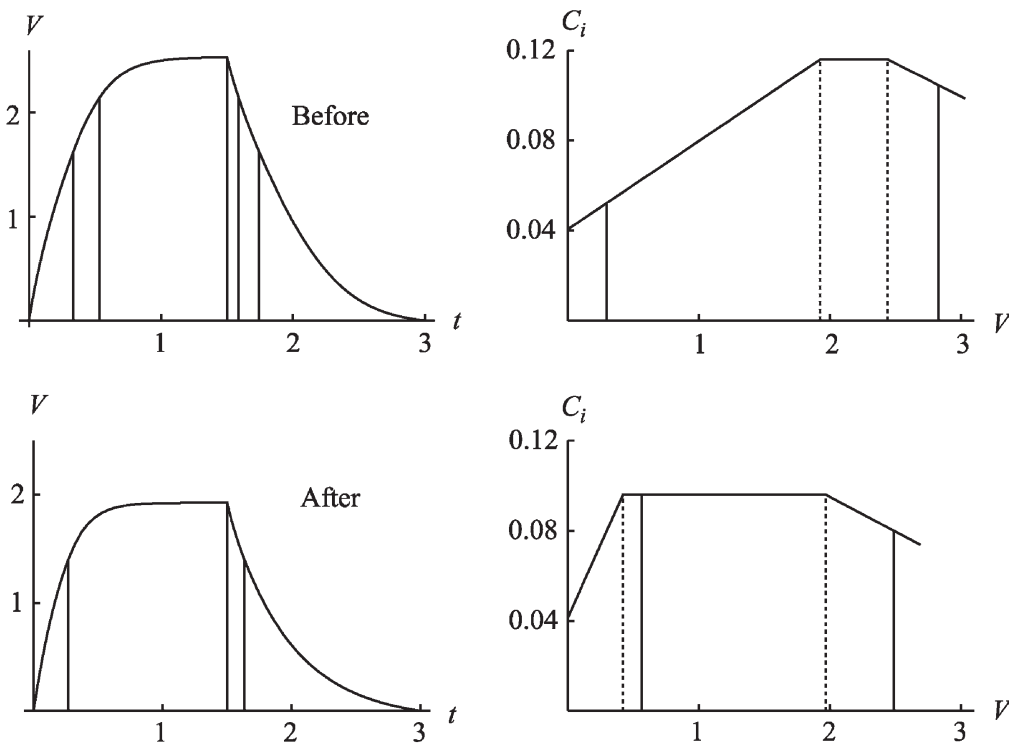

FIGURE 9 Volume and compliance functions before and after recruitment maneuver for patient 2. 
TABLE III Tidal Volumes, end-expiratory pressures, mean alveolar pressures, and average volume for 14 patients before and after recruitment maneuver, using data from Svantesson et al. (1998)

\begin{tabular}{|c|c|c|c|c|c|c|c|c|}
\hline \multirow[b]{2}{*}{ Patient no } & \multicolumn{4}{|c|}{ Before recruitment } & \multicolumn{4}{|c|}{ After recruitment } \\
\hline & $V_{T}$ & $P_{\mathrm{ex}}$ & $P_{m}$ & $V_{\text {ave }}$ & $V_{T}$ & $P_{\mathrm{ex}}$ & $P_{m}$ & $V_{\text {ave }}$ \\
\hline 1 & 1.730 & 5.292 & 18.155 & 0.953 & 1.508 & 5.644 & 15.235 & 0.877 \\
\hline 2 & 2.534 & 5.708 & 19.141 & 1.393 & 1.925 & 5.900 & 16.991 & 1.095 \\
\hline 3 & 2.104 & 5.552 & 18.873 & 1.148 & 1.817 & 5.622 & 17.333 & 1.047 \\
\hline 4 & 2.400 & 6.285 & 18.800 & 1.346 & 2.438 & 6.775 & 18.278 & 1.386 \\
\hline 5 & 2.832 & 7.364 & 18.662 & 1.603 & 2.462 & 7.374 & 16.775 & 1.415 \\
\hline 6 & 2.456 & 6.009 & 19.264 & 1.344 & 2.328 & 6.455 & 18.410 & 1.321 \\
\hline 7 & 3.490 & 7.953 & 20.501 & 1.874 & 2.629 & 8.290 & 15.465 & 1.541 \\
\hline 8 & 3.187 & 7.518 & 19.684 & 1.735 & 2.438 & 7.275 & 16.919 & 1.422 \\
\hline 10 & 3.591 & 10.231 & 19.886 & 1.986 & 2.734 & 8.472 & 16.206 & 1.584 \\
\hline 11 & 3.281 & 9.781 & 17.637 & 1.838 & 3.055 & 9.079 & 17.723 & 1.723 \\
\hline 12 & 2.853 & 7.152 & 19.165 & 1.577 & 2.308 & 7.321 & 16.680 & 1.352 \\
\hline 13 & 2.820 & 6.808 & 19.009 & 1.570 & 1.844 & 6.397 & 14.673 & 1.090 \\
\hline 14 & 3.153 & 7.936 & 20.067 & 1.707 & 2.589 & 7.726 & 16.547 & 1.504 \\
\hline 15 & 2.948 & 7.418 & 19.432 & 1.681 & 1.944 & 6.224 & 16.676 & 1.113 \\
\hline Average & 2.902 & 7.251 & 19.186 & 1.623 & 2.340 & 7.106 & 16.789 & 1.355 \\
\hline
\end{tabular}

increase, both before and after recruitment. The percentage changes in the key outcome variable before and after recruitment are much smaller at the lower PEEPs. Another aspect of the change with PEEP before and after the recruitment maneuvers is which segments of the compliance function is the ventilation taking place. For the data in Table IV, all three segments of $C_{i}(V)$ are used for $P_{\text {peep }}=0,2,4$, before and after recruitment. For $P_{\text {peep }}=6,8,10,12$, only the second and third segments are used for the after recruitment compliance function. For $P_{\text {peep }}=15$, the second and third segments are used before and after recruitment. This would indicate that following recruitment, the lower inflection point would not be crossed for $P_{\text {peep }} \geq 6 \mathrm{~cm} \mathrm{H}_{2} \mathrm{O}$. In this individual after recruitment, ventilation could take place above the lower inflection point at lower PEEP levels and hence, lower mean alveolar pressures, with similar average volumes to those found before recruitment.

In simulations performed for the other patients, there is even more variability. For example, using the compliance data for patient 2, the same PEEP simulations were performed. It was found that for $P_{\text {peep }} \geq 4 \mathrm{~cm} \mathrm{H}_{2} \mathrm{O}$ after recruitment, the lower inflection point is avoided. At $P_{\text {peep }}=4$, the mean alveolar pressure was lower by $10.3 \%$ by the recruitment maneuver. The importance of a mathematical model is evident in this type of theoretical approach. For the clinician it is a balancing act to choose values for PEEP that lead to sufficient oxygenation levels and non-collapsing alveoli without the imposing high internal pressures.

\section{CLINICAL IMPLICATIONS}

Recent experimental and clinical evidence indicates that high pressure mechanical ventilation-at volumes above the upper inflection point-can inflict lung damage. Pressure limiting ventilatory strategies have been shown to lower mortality in ARDS. Ventilation with a pattern in which the system repeatedly traverses the lower inflection point is also observed in experimental models of lung injury, although the current clinical evidence is somewhat less compelling. Accordingly, current ventilatory strategies endeavor to avoid overdistention, while avoiding repetitive cycles of recruitment-derecruitment. In this context, pressure targeted ventilation with high applied PEEP provides a valuable adjunct, for it absolutely restricts the maximal alveolar pressure. Although pressure control has the salutary effect of limiting

TABLE IV Tidal Volumes, total PEEPs, mean alveolar pressures, and average volumes for different levels of applied PEEP using the compliance functions parameters for patient 1 in Svantesson et al. (1998)

\begin{tabular}{|c|c|c|c|c|c|c|c|c|}
\hline \multirow[b]{2}{*}{ PEEP } & \multicolumn{4}{|c|}{ Before recruitment } & \multicolumn{4}{|c|}{ After recruitment } \\
\hline & $V_{T}$ & $P_{\mathrm{ex}}$ & $P_{m}$ & $V_{\text {ave }}$ & $V_{T}$ & $P_{\mathrm{ex}}$ & $P_{m}$ & $V_{\text {ave }}$ \\
\hline 0.0 & 2.066 & 0.258 & 16.351 & 1.134 & 1.925 & 0.405 & 16.114 & 1.122 \\
\hline 2.0 & 1.935 & 2.273 & 17.066 & 1.063 & 1.798 & 2.530 & 16.108 & 1.047 \\
\hline 4.0 & 1.800 & 4.286 & 17.791 & 0.990 & 1.623 & 4.640 & 15.728 & 0.945 \\
\hline 6.0 & 1.660 & 6.297 & 18.517 & 0.915 & 1.407 & 6.597 & 15.050 & 0.818 \\
\hline 8.0 & 1.517 & 8.305 & 19.231 & 0.837 & 1.256 & 8.518 & 15.503 & 0.728 \\
\hline 10.0 & 1.371 & 10.306 & 19.929 & 0.757 & 1.108 & 10.439 & 16.146 & 0.640 \\
\hline 12.0 & 1.222 & 12.298 & 20.593 & 0.675 & 0.963 & 12.358 & 16.945 & 0.553 \\
\hline 15.0 & 1.005 & 15.252 & 21.708 & 0.555 & 0.768 & 15.234 & 18.504 & 0.434 \\
\hline
\end{tabular}


alveolar pressures, in choosing this mode of ventilation, the clinician sacrifices control over the level of ventilation i.e. the tidal volume or average lung volume.

Desired outcomes of mechanical ventilation are a minute ventilation $\left(\dot{V}_{E}=f V_{T}\right)$, adequate to protect the systemic $\mathrm{pH}$ (via removal of $\mathrm{CO}_{2}$ ), and a mean alveolar pressure which is sufficient to maintain lung volume and support adequate oxygenation. Predictions of the threesegment model differ from those of the constant compliance model in a number of clinically relevant respects.

In the constant compliance model, tidal volume diminishes with increasing respiratory frequency; the decline arises from the effects of shortened inspiratory time (less opportunity for alveolar filling) as well as increasing total PEEP (decreased gradient of driving pressure). The three-segment model predicts a smaller dependence of tidal volume on ventilatory frequency. This is potentially important in the clinical setting, as it suggests that increasing respiratory frequency in this pressure-limited mode of ventilation may preserve tidal volume and increase minute ventilation more than expected from the constant compliance case. As the maximum acceptable driving pressure may be limited by the relatively small difference between a high applied PEEP (used to avoid derecruitment) and a lower $P_{\text {set }}$ (to avoid overdistension), increased respiratory frequency may be the only mechanism for elevating minute ventilation.

Similarly, in a constant compliance system with airflow obstruction, there is a strong dependence of tidal volume on inspiratory time fraction $D$, with a clear optimum (Marini and Crooke, 1993). Elevating the inspiratory time fraction above the optimal value leads to a rapid decline in $V_{T}$. The dependence of tidal volume on the duty cycle in the three segment model is somewhat less marked, indicating that reasonable increases in duty cycle to increase mean alveolar pressure (vide infra) may not overtly compromise minute ventilation.

Our model does not attempt to address the microstructural basis for observed nonlinearity of the $P_{\text {elastic }}-$ $V_{\text {curve }}$. Rather, it addresses the effects of a nonlinear macroscopic pressure-volume curve behavior on common clinical outcomes: tidal volume, end-expiratory pressure, mean alveolar pressure, and average lung volume. In this regard, it predicts important consequences arising from the presence of nonlinearity. Although the regional distribution of pressure and tidal volume are also of crucial importance, our data indicate that the presence of upper and lower inflection points alone will significantly affect the macroscopic outcomes most commonly tracked in the clinical setting. The qualitative behaviors are those of importance in this setting, as they suggest the overall responses to common clinical manipulations of ventilator settings. Better knowledge of these effects may be useful in optimizing ventilatory strategies in the clinical setting.

\section{CONCLUSION}

In this paper, a mathematical model for pressure controlled ventilation with three-segment compliance function has been presented and used to study the effects of clinician-set inputs on the key ventilatory outcome variables. The model can be used to predict the effects on the outcome variables using recruitment maneuvers.

\section{Acknowledgements}

The authors thank the referees for their suggestions on substance and style.

\section{References}

Amato, M.B., et al. (1998) "Effect of a protective-ventilatory strategy on mortality in the acute respiratory distress syndrome", N. Engl. J. Med. 338, 347-354.

Barbini, P. (1982) "Non-linear model of the mechanics of breathing applied to the use and design of ventilators", J. Biomed. Eng. 4, 204-304.

Burke, W.C., et al. (1993) "Comparison of mathematical and mechanical models of pressure-controlled ventilation", J. Appl. Physiol. 74, 922-933.

Chandra, A., et al. (1993) "A flow-based mathematical model of respiratory dynamics", Am. Rev. Respir. Dis. 25, A879.

Crooke, P.S. and Marini, J.J. (1993) "A nonlinear mathematical model of pressure preset ventilation: description and limiting values for key outcome variables", Math. Mod. Meth. Appl. Sci. 3, 839-859.

Crooke, P.S., Head, J.D. and Marini, J.J. (1996) "A general twocompartment model for mechanical ventilation", Math. Comput. Model. 24, 1-18.

Fujino, Y., Goddon, S., et al. (2001) "Repetitive high-pressure recruitment maneuvers required to maximally recruit lung in a sheep model of acute respiratory distress syndrome", Crit. Care Med. 29, 1579-1586.

Gattioni, L., Pelosi, P., Crotti, S. and Valenza, F. (1995) "Effects of positive end-expiratory pressure on regional distribution of tidal volume and recruitment in adult respiratory distress syndrome", $\mathrm{Am}$. J. Respir. Crit. Care Med. 151, 1807-1814.

Hickling, K.G. (1998) "The pressure-volume curve is greatly modified by recruitment", Am. J. Respir. Crit. Care Med. 158, 194-202.

Hickling, K.G. (2001) "Best compliance during a decremental, but not incremental, positive end-expiratory pressure trial is related to openlung positive end-expiratory pressure", Am. J. Respir. Crit. Care Med. 163, 69-78.

Hotchkiss, J.R., Crooke, P.S., Adams, A.B. and Marini, J.J. (1994) "Implications of a biphasic two-compartment model of constant flow ventilation for the clinical setting", J. Crit. Care 9, 114-123.

Hotchkiss, J.R., Crooke, P.S. and Marini, J.J. (1996) "Theoretical interactions between ventilator settings and proximal dead space ventilation during tracheal gas insufflation", Intensive Care Med. 22, $1112-1119$.

Jonson, B. and Svantesson, C. (1999) "Elastic pressure-volume curves: what information do they convey?", Thorax 54, 82-87.

Jonson, B., Richard, J., et al. (1999) "Pressure-volume curves and compliance in acute lung injury", Am. J. Respir. Crit. Care Med. 159, $1172-1178$.

Laghi, F., et al. (1999) "Influence of ventilator settings in determining respiratory frequency during mechanical ventilation", Am. J. Respir. Crit. Care Med. 160, 1766-1770.

Maggiore, S.M., Jonson, B., et al. (2001) "Alveolar derecruitment at decremental positive end-expiratory pressure levels in acute lung injury", Am. J. Respir. Crit. Care Med. 164, 795-801.

Mancini, M., Zavala, E., et al. (2001) "Mechanisms of pulmonary gas exchange improvement during protective ventilatory strategy in acute respiratory distress syndrome", Am. J. Respir. Crit. Care Med. 164, $1448-1453$.

Marini, J.J. and Crooke, P.S. (1993) "A general mathematical model for respiratory dynamics relevant to the clinical setting", Am. Rev. Respir. Dis. 147, 14-24. 
Marini, J.J., Crooke, P.S. and Truwit, J.D. (1989) "Determinants and limits of pressure-preset ventilation: a mathematical model of pressure control", J. Appl. Physiol. 67, 1081-1092.

Mols, G., Brandes, I., et al. (1999) "Volume-dependent compliance in ARDS: proposal of a new diagnostic concept", Intensive Care Med. 25, 1084-1091.

Mols, G., Hermle, G., et al. (2001) "Volume-dependent compliance and ventilation-perfusion mismatch in surfactant-depleted isolated rabbit lungs", Crit. Care Med. 29, 144-151.

Morgenstern, U. and Kaiser, S. (1995) "Mathematical modelling of ventilation mechanics", Int. J. Clin. Monit. Comput. 12, 105-112.

Nikischin, W., Gerhardt, T., et al. (1998) "A new method to analyze lung compliance when pressure-volume relationship is nonlinear", $\mathrm{Am}$. J. Respir. Crit. Care Med. 158, 1052-1060.

Papadakos, P.J. and Lachmann, B. (2002) "The open lung concept of alveolar recruitment can improve outcome in respiratory failure and ARDS", Mt. Sinai J. Med. 69, 73-77.

Servillo, G., et al. (1997) "Pressure-volume curves in acute respiratory failure: automated low flow inflation versus occlusion", Am. J. Respir Crit. Care Med. 155, 1629-1636.
Silva, N., et al. (1992) "Nonlinear pressure/volume relationship and measurements of lung mechanics in infants", Pediatr. Pulmonol. 12, $146-512$.

Suki, B., et al. (1998) "Mathematical modeling of the first inflation of degassed lungs", Ann. Biomed. Eng. 26, 608-617.

Svantesson, C., Sigurdsson, S., et al. (1998) "Effects of recruitment of collapsed lung units on the elastic pressure-volume relationship in anesthetized healthy adults", Acta Anaesthesiol. Scand. 42, $1149-1156$.

Venegas, J.G., Harris, R.S. and Simon, B.A. (1998) "A comprehensive equation for the pulmonary pressure-volume curve", J. Appl. Physiol. 84, 389-395.

Villagra, A., et al. (2002) "Recruitment maneuvers during lung protective ventilation in acute respiratory distress syndrome", Am. J. Respir. Crit. Care Med. 165, 165-170.

Wald, A.A., Murphy, T.W. and Mazzia, V.D. (1968) "A theoretical study of controlled ventilation", IEEE Trans. Biomed. Eng. 15, 237-248.

Yap, D.Y.K., et al. (1994) "Influences of parenchyma tethering on the reopening of closed pulmonary airways", J. Appl. Physiol. 76, 2095-2105. 


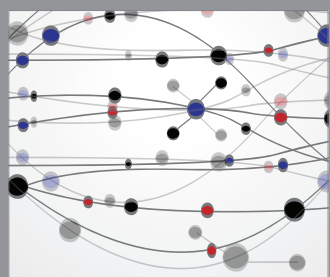

The Scientific World Journal
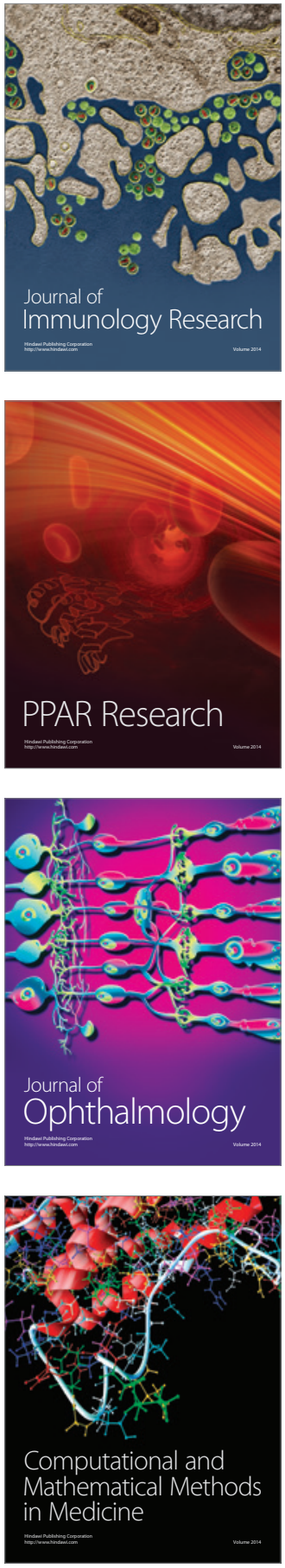

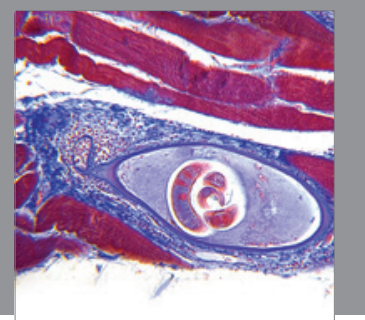

Gastroenterology

Research and Practice
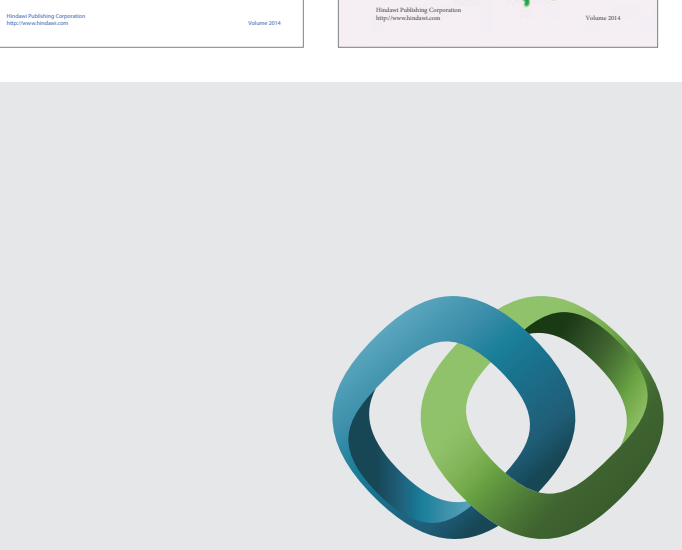

\section{Hindawi}

Submit your manuscripts at

http://www.hindawi.com
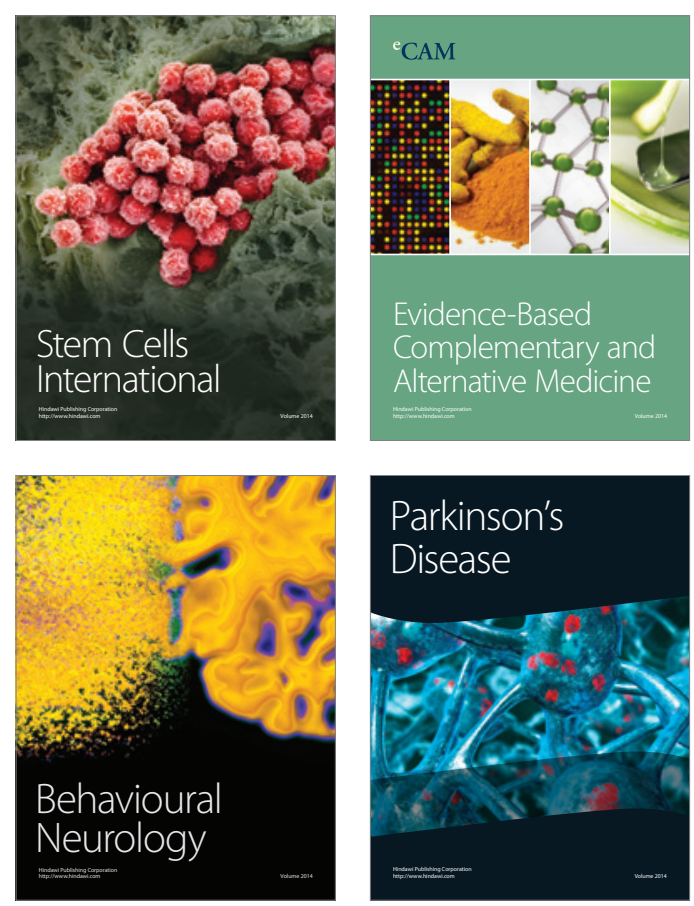

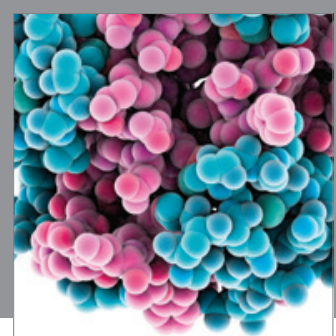

Journal of
Diabetes Research

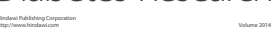

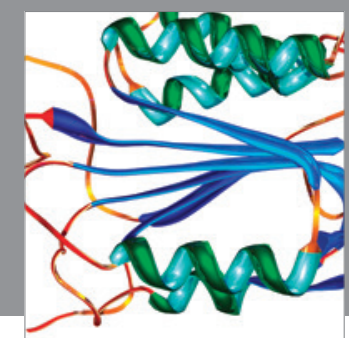

Disease Markers
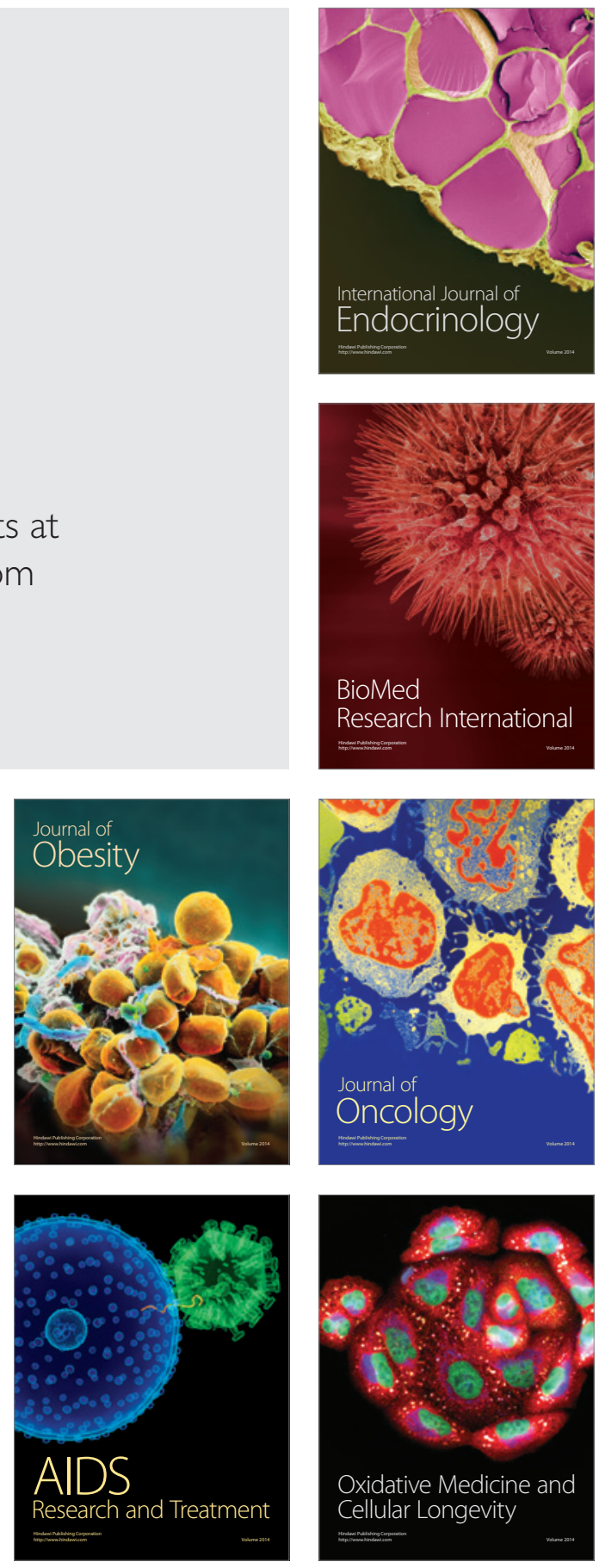